\title{
PA-057 ENHANCING TUBERCULOSIS DETECTION BY TRAINED RATS AND TRACKING OF MISSED PATIENTS THROUGH COMMUNITY-BASED STRATEGY IN TB HIGH-BURDEN COUNTRIES
}

Georgies Mgode, ${ }_{1}^{1}$ Christophe Cox ${ }_{1}^{2}$ Dickens Bwana, ${ }_{1}{ }^{3}$ Leah Mtui, ${ }^{4}$ Daniel Magesa, ${ }_{1}^{4}$ Amos Kahwa, ${ }^{5}$ Godfrey Mfinanga, ${ }^{6}$ Christiaan Mulder ${ }^{7}$ ' ${ }^{1}$ Sokoine University of Agriculture, Tanzania; ${ }^{2}$ APOPO project, Tanzania; ${ }^{3}$ MUKIKUTE, Tanzania; ${ }^{4}$ PASADA, Tanzania; ${ }^{5}$ NIMR Muhimbili, Tanzania; ${ }^{6}$ NIMR, Tanzania; ${ }^{7}$ APOPO project, The Netherlands

\subsection{6/bmjgh-2016-000260.90}

Background Tuberculosis (TB) diagnosis in most sub-Saharan African countries with high TB burden is by direct smear microscopy that has low sensitivity. More sensitive tests like GeneXpert are expensive and not yet available in most African settings. Therefore, a need for cheap and rapid tests is inevitable. Trained rats (Cricetomys spp.) detect TB by targeting the Mycobacterium tuberculosis specific volatile compounds. Accredited rats evaluated 150 sputa in 20 minutes. We report on intervention involving TB detection by rats and additional patient tracking by community healthcare workers from MUKIKUTE and PASADA for treatment.

Methods Sputum was collected in hospitals in Dar es Salaam, Coast and Morogoro, Tanzania after microscopy. Sputa were heat inactivated at $100 \mathrm{oC} \times 30$ minutes to kill pathogens and thereafter presented to rats in random computer generated positions for evaluation. Samples indicated as TB-positive by rats were confirmed by concentrated smear microscopy whether they contained TB bacilli. Confirmed TB cases were reported to community healthcare workers and hospitals for tracking and treatment. Healthcare workers recorded contact details of presumptive TB patients for subsequent tracking when detected by rats.

Results From 2011 to 2015 detection rats evaluated 306,346 sputum samples from 152,118 presumptive TB patients, which 
were also tested by microscopy in hospitals. DOTS smear-positive TB patients were 21,911 and rats detected an additional 7961 patients missed in hospitals. Community healthcare workers tracking the additional patients brought 2715 additional TB patients to treatment whereas treatment initiation increased from 56\% (1020/ $1812)$ in 2013 to $73 \%(870 / 1,198)$ in 2015 . This prevented TB transmission to 27,150 to 40,725 people since one untreated TB patient can spread the disease to $10-15$ persons annually.

Conclusions TB detection using rats and involvement of community healthcare workers to track the additional TB patients for treatment can increase TB detection and treatment initiation rate of missed TB cases. 